\title{
SATISFAÇÃO DOS FAMILIARES COM A HUMANIZAÇÃO DA ASSISTÊNCIA EM UTI
}

FAMILY MEMBERS' SATISFACTION WITH HUMANIZATION OF CARE AT THE ICU

SATISFACCIÓN DE LOS FAMILIARES CON LA HUMANIZACIÓN DE LA ATENCIÓN EN UCI

Elisângela de Jesus Macêdo Araújo ${ }^{1}$

Keila Maria de Azevedo Ponte ${ }^{2}$

Lívia Mara de Araújo 3

Maria Sinara Farias ${ }^{4}$

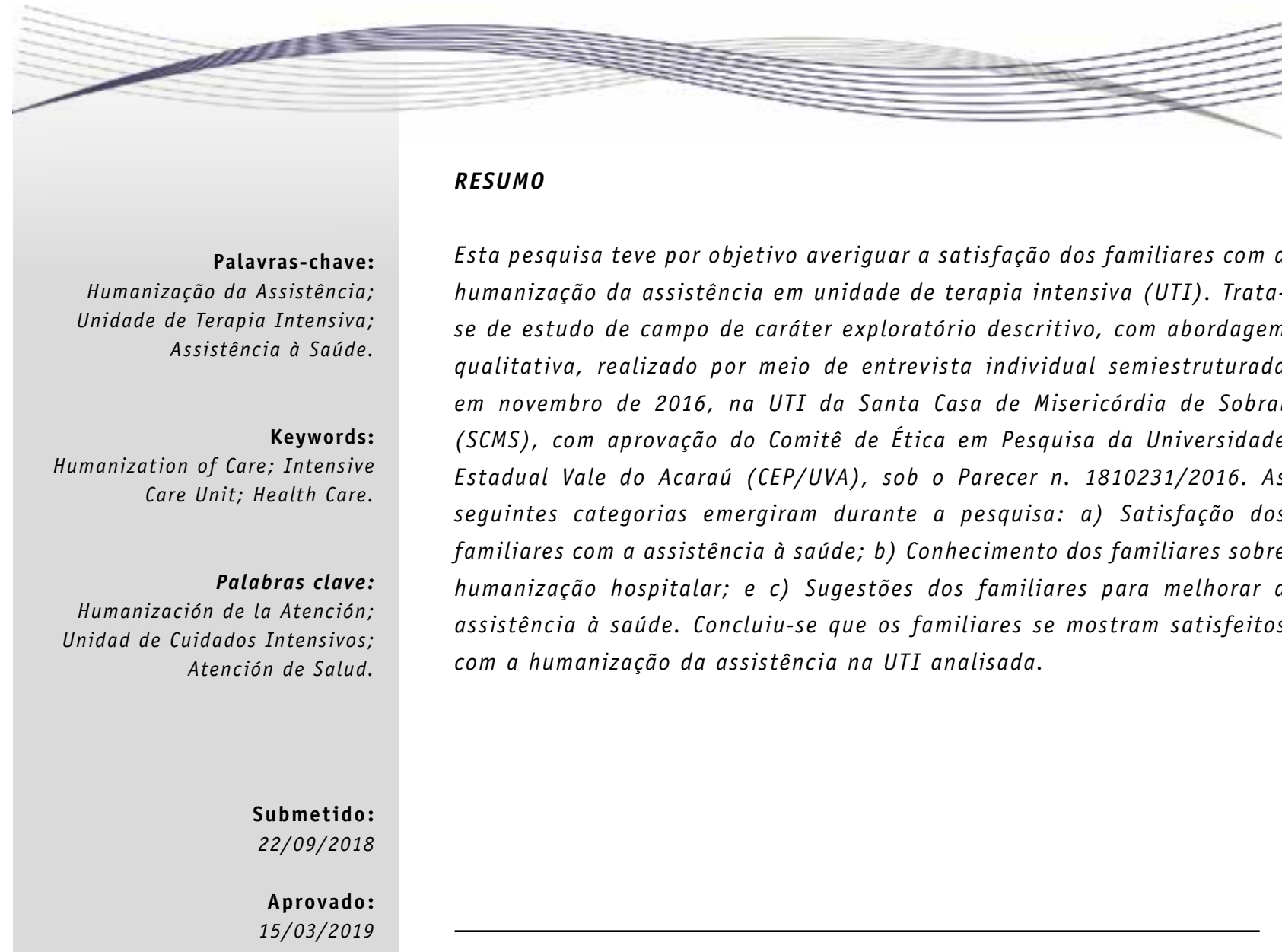

Autor(a) para Correspondência: Elisângela de Jesus Macêdo Araújo R. Prof. Manoel Pinto Filho, No 1077 - Padre Ibiapina Sobral (CE) - CEP: 62023-080 E-mail:eli_araujohc@hotmail.com

1. Enfermeira graduada pelo Centro Universitário Instituto Superior de Teologia Aplicada (UnInta). Professora no Instituto Centro de Ensino Tecnológico (CENTEC). Sobral (CE), Brasil.

2. Enfermeira. Professora no Curso de Graduação em Enfermagem da Universidade Estadual Vale do Acaraú (UVA). Sobral (CE), Brasil.

3. Enfermeira graduada pelo UnInta. Sobral (CE), Brasil.

4. Enfermeira. Aluna de Doutorado em Cuidados Clínicos de Enfermagem e Saúde na Universidade Estadual do Ceará (UECE). Fortaleza (CE), Brasil. 


\section{ABSTRACT}

This research aimed to investigate family members' satisfaction with humanization of care at the intensive care unit (ICU). This is a descriptive exploratory field study, with a qualitative approach, conducted by means of a semi-structured individual interview in November 2016, at the ICU of the Holy House of Mercy Hospital in Sobral (Santa Casa de Misericórdia de Sobral - SCMS), after approval by the Research Ethics Committee of the Ceará State University Vale do Acaraú (REC/Universidade Estadual Vale do Acaraú - UVA), under the Advisory Opinion No. 1810231/2016. The following categories emerged during the research: a) Family members' satisfaction with health care; b) Family members' knowledge on hospital humanization; and c) Family members' suggestions to improve health care. It was concluded that family members showed to be satisfied with humanization of care at the ICU under analysis.

\section{RESUMEN}

Esta investigación tuvo como objetivo investigar la satisfacción de los familiares con la humanización de la atención en unidad de cuidados intensivos (UCI). Este es un estudio de campo exploratorio descriptivo, con enfoque cualitativo, realizado mediante entrevista individual semi-estructurada en noviembre de 2016, en la UCI del Hospital de la Santa Casa de la Misericordia de Sobral (Santa Casa de Misericórdia de Sobral - SCMS), con aprobación del Comité de Ética en Investigación de la Universidad del Estado de Ceará Vale do Acaraú (CEI/ Universidade Estadual Vale do Acaraú - UVA), bajo la Opinión No. 1810231/2016. Las siguientes categorías surgieron durante la investigación: a) Satisfacción de los familiares con la atención de salud; b) Conocimiento de los familiares acerca de la humanización hospitalaria; y c) Sugerencias de los familiares para mejorar la atención de salud. Se concluyó que los familiares se muestran satisfechos con la humanización de la atención en la UCI bajo análisis.

\section{INTRODUÇÃO}

Cuidar do humano é um ato que exprime a necessidade do encontro de duas pessoas em certa situação ou determinado ambiente. Nessa perspectiva, os cuidados de enfermagem prestados ao paciente de modo objetivo, mediante técnicas e procedimentos padronizados, também se pautam por uma vertente subjetiva, balizada por sensibilidade, criatividade e intuição ${ }^{1}$.

Em tal contexto, o enfermeiro é o profissional responsável por coordenar e gerenciar o processo de assistência à saúde a ser desenvolvido com o paciente e tudo o que está envolvido no contexto hospitalar. A equipe de enfermagem desempenha um importante papel no cuidado direto ao paciente, por exemplo, na realização de procedimentos ${ }^{2}$. 0 paciente, com suas especificidades e necessidades com vistas à recuperação ou alta, constitui a principal razão da assistência à saúde - que pressupõe eficácia e eficiência, com comprometimento daqueles que dela participam, garantindo a qualidade do cuidado e, principalmente, a satisfação do paciente e de seus familiares ${ }^{3}$.

0 paciente que apresenta dependência da assistência à saúde, como é o caso da pessoa internada em unidade de terapia intensiva (UTI), recorre aos cuidados de familiares e da equipe de saúde - onde os profissionais de enfermagem assumem destaque.

A UTI é um ambiente hospitalar destinado a casos graves, pacientes que apresentam quadro clínico recuperável ou não. Esse cenário envolve procedimentos invasivos e risco de morte, podendo mostrar-se hostil e desalentador. Trata-se de um local onde atuam profissionais das mais variadas áreas da saúde, principalmente os profissionais de enfermagem, responsáveis por grande parte das atividades do cuidado intensivo, como: a) realização de procedimentos; b) monitorização constante; c) uso de tecnologia aplicada à saúde etc. ${ }^{4}$.

Diante dessa atmosfera, criou-se a Política Nacional de Humanização (PNH), com o propósito de demonstrar que o Sistema Único de Saúde (SUS) está pronto para fazer frente aos desafios e problemas relativos à efetivação da $\mathrm{PNH}$, os quais têm sido enfaticamente relatados por profissionais da saúde que apontam falta de condições para alcançar os objetivos almejados. Ressalta-se, ainda, que a humanização da assistência abrange circunstâncias sociais, éticas, educacionais e psíquicas dos relacionamentos humanos, que têm origem no âmbito da família 5 .

A família constitui o pilar fundamental das sociedades - trata-se da base sobre a qual a maioria 
dos seres humanos constrói sua personalidade. Essa célula primária apresenta valor inalienável, como reconhece a Declaração Universal dos Direitos Humanos (DUDH), que atesta sua importância no desenvolvimento dos indivíduos. Por isso, é essencial definir e consolidar um caminho para integrar a família aos cuidados hospitalares ${ }^{6}$.

Tendo em vista o panorama apresentado, esta pesquisa buscou averiguar a satisfação dos familiares com a humanização da assistência em UTI.

\section{METODOLOGIA}

Trata-se de estudo exploratório descritivo, com abordagem qualitativa, realizado na UTI da Santa Casa de Misericórdia de Sobral (SCMS), hospital de referência no norte do Ceará. Os dados foram coletados em novembro de 2016, com 10 familiares de pacientes internados nessa UTI.

Os critérios de inclusão adotados foram: a) familiares com primeiro, segundo ou terceiro grau de parentesco; e b) em caso de constante ausência de familiares, cuidadores que visitavam os pacientes com frequência e que se apresentavam como responsáveis por eles. Já o critério de exclusão foi: familiares menores de 18 anos.

0 s dados foram coletados por meio de entrevista semiestruturada, recorrendo às seguintes perguntas norteadoras:

- Qual é a satisfação dos familiares com a humanização da assistência em UTI?

- Qual é o conhecimento dos familiares sobre humanização hospitalar?

- Quais são as sugestões dos familiares para melhorar a assistência à saúde?

Os dados foram submetidos à técnica de análise de conteúdo temática ${ }^{7}$, com as seguintes etapas: a) fase exploratória, na qual se amadurece o objeto de estudo e se delimita o problema de investigação; b) coleta de dados, em que se recolhem informações para fazer frente ao problema de pesquisa; e c) análise de dados, com tratamento, por inferência e interpretação, dos dados obtidos.

Esta pesquisa - aprovada pelo Comitê de Ética em Pesquisa da Universidade Estadual Vale do Acaraú (CEP/UVA), sob o Parecer n. 1810231/2016 - cumpriu todos os princípios éticos da Resolução do Conselho Nacional de Saúde (CNS) n. 466/2012, que dispõe sobre as diretrizes e normas regulamentadoras de pesquisas envolvendo seres humanos.

\section{RESULTADOS E DISCUSSÃO}

Após a coleta dos dados, as informações foram analisadas e classificadas em categorias, a saber: a) Caracterização dos participantes; b) Satisfação dos familiares com a prática assistencial; e c) Conhecimento dos familiares sobre humanização hospitalar.

\section{Caracterização dos participantes}

Participaram deste estudo 10 familiares de pacientes internados em UTI há 72 horas ou mais. Houve $40 \%$ de participantes do sexo masculino e $60 \%$ do sexo feminino. No sexo masculino, a idade variou dos 29 anos (50\%) aos 66 anos (25\%); já no sexo feminino, a idade foi dos 25 anos $(16,6 \%)$ aos 45 anos $(16,6 \%)$.

No tocante à procedência, há representação de diversas localidades do Ceará, com prevalência de municípios do norte do estado. Quanto ao grau de parentesco, a distribuição entre primeiro, segundo e terceiro graus é equivalente ( 1 filho; 2 primas; 3 irmãos; 1 mãe; 1 tio; 1 esposa; e 1 sobrinha).

No que concerne ao grau de escolaridade, $10 \%$ dos participantes eram analfabetos, 30\% tinham Ensino Fundamental incompleto, 30\% tinham Ensino Médio incompleto e $30 \%$ tinham Ensino Médio completo. Quanto à religião, $20 \%$ eram evangélicos e $80 \%$ eram católicos. Considerando a profissão, $20 \%$ eram agricultores, $20 \%$ eram do lar, $10 \%$ eram porteiros, $20 \%$ eram auxiliares de produção, $10 \%$ eram cabeleireiros, $10 \%$ eram cozinheiras e $10 \%$ eram diaristas.

Ter um familiar internado em UTI causa anseio por informações detalhadas e contato direto com o paciente, sendo necessário o deslocamento até - local, em busca de conforto e esperança; o que mais importa é ver o familiar, tocá-lo e saber como vai a assistência da equipe de saúde, uma vez que

\section{...é essencial definir e consolidar um caminho para integrar a família aos cuidados hospitalares...}


os cuidados são delegados a profissionais que lidam com uma situação de vulnerabilidade.

Nesse cenário, durante a visita, observa-se o envolvimento familiar no âmbito hospitalar. A internação de um parente tende a desestruturar a família e é necessário que todos contribuam para alcançar um objetivo comum: restabelecer a saúde desse ente querido. Nesse momento, os membros da família tendem a se sensibilizar e apoiar um ao outro - observa-se a organização familiar em meio a dor, sofrimento, aflição e angústia, dentre outros sentimentos vivenciados de modo diferente por cada indivíduo.

Diante do exposto, constata-se que tanto a família quanto o paciente vivenciam extrema vulnerabilidade, com reduzido contato entre parentes, deixando a assistência à saúde aos cuidados de uma equipe multidisciplinar intensivista.

Assim, é a família que recebe suporte material e psicológico tanto em situações de maior estresse quanto na alternância entre saúde/doença, alegria/ tristeza, esperança/desespero etc. ${ }^{6}$.

\section{Satisfação dos familiares com a assistência à saúde}

Durante a entrevista, percebeu-se a satisfação dos familiares diante da assistência à saúde - cuja qualidade relatam ser excelente ou boa.

As falas abaixo ilustram a satisfação dos familiares com a assistência à saúde:

No momento, para mim está excelente, né? Porque já tive notícia, pela primeira vez que estou vindo aqui, mas já estou tendo notícia que ele está bem melhor, que os médicos estão fazendo o que podem para salvar a vida dele, e isso para nós já é excelente, estamos tendo um bom retorno aqui do hospital, isso já é o suficiente, um bom atendimento. (Familiar 01)

É um bom atendimento, né? A assistência que a gente recebeu é tudo que a gente precisa e é nesses momentos que a gente aprende cada vez mais que precisa um do outro. (Familiar 05)

Ao discorrer sobre a assistência à saúde, os familiares mencionam o médico com frequência, apresentando-o como principal integrante da equipe de saúde e responsável pela recuperação do

\section{...o único momento reservado ao contato dos familiares com os pacientes é o limitado per iodo de visita...}

paciente. As falas evidenciam que a enfermagem fica esquecida, apesar de sua crucial contribuição para o sucesso do tratamento e da recuperação do paciente; isso se deve à falta de diálogo aberto entre os profissionais de enfermagem e a família, que esquece de mencioná-los em suas falas por desconhecer o papel dessa categoria na assistência à saúde.

Tal fato reforça a necessidade de ações de educação em saúde, de modo que as informações pertinentes sejam oferecidas pelos profissionais no momento da visita, de forma clara e de fácil compreensão, o que requer dos profissionais da saúde habilidade de comunicação e compreensão da relação terapêutica no contato entre paciente, família e equipe de saúde.

Diante disso, percebe-se a necessidade e importância de avaliar a eficácia e eficiência da assistência à saúde junto com os usuários. A partir dessa avaliação, pode-se planejar mudanças nos procedimentos e nas condutas tanto da gerência do serviço como na equipe operacional - as atitudes são fatores que contribuem para a humanização da assistência tanto por parte dos profissionais da saúde quanto dos familiares que visitam seu ente querido. A enfermagem, categoria eminentemente cuidadora, é responsável por promover autonomia, equilíbrio, tranquilidade e bem-estar entre os pacientes e os demais usuários dos serviços disponíveis.

Assim, o único momento reservado ao contato dos familiares com os pacientes é o limitado período de visita - e é aí que os familiares "despejam" seus anseios e suas emoções, suas dúvidas quanto à doença, suas preocupações e reflexões sobre 0 impacto da internação nos papéis sociais. Em virtude disso, as informações fornecidas pelos profissionais da saúde no período de visita, geralmente por parte de médicos e enfermeiros, devem ser claras e empáticas ${ }^{8}$. 
Nesse contexto, a satisfação dos familiares é vital na avaliação da qualidade da assistência à saúde identificá-la é uma responsabilidade essencial dos profissionais que atuam em UTI ${ }^{9}$.

Nessa perspectiva, vale salientar que a comunicação é a pedra de toque na relação entre enfermeiro e usuário estabelecida na prática assistencial. Vivenciar a internação de um parente em UTI requer dos familiares a capacidade de compreender seus próprios sentimentos e elaborar estratégias para o enfrentamento do problema. Nesse momento se observa a necessidade do acolhimento, de repassar informações aos familiares confortandoos durante o horário de visita ${ }^{8}$.

\section{Conhecimento dos familiares sobre humanização hospitalar}

A humanização da assistência visa a garantir a autonomia e dignidade do paciente, assim, os profissionais da saúde o tratam com respeito, carinho e amor, respeitando seus limites, oferecendo orientações sobre sua patologia e os procedimentos adotados e cuidando não só da doença, mas da pessoa - o que demanda um olhar diferenciado.

Tal aspecto é evidenciado nessa categoria, como ilustram as seguintes falas dos familiares:

Não sei bem o foco, mas eu acho, assim, que pode ser a questão do atendimento, atenção, cuidado, eles são muito caridosos, fazem o que podem para controlar os familiares. (Familiar 02)

Pra mim, ali dentro está superlimpo eles estão cuidando do meu irmão, o que eu entendo é isso. (Familiar 03)

Para falar a verdade, eu não entendo quase nada, apesar de saber que é muito importante, mas eu não entendo quase nada, dificilmente tem alguém meu no hospital. (Familiar 05)

No tocante à categoria Conhecimento dos familiares sobre humanização hospitalar, ressalta-se que esse processo se relaciona a uma forma peculiar no trato com o ser humano. Nos hospitais públicos, esse processo se mostra ainda mais necessário, tendo em vista a necessidade dos usuários do SUS e as precariedades vivenciadas por eles ${ }^{10}$.

Nessa categoria, constata-se deficiência de conhecimento sobre humanização hospitalar

\section{humanização hospitalar, ressalta-se que esse processo se relaciona a uma forma peculiar no trato com o ser humano.}

entre os familiares, um aspecto importante que eles associam a caridade, cordialidade, bom atendimento, acolhimento e cuidado ao paciente, ressaltando, ainda, a limpeza do local. Nesse momento, faz-se necessário trabalhar a humanização entre profissionais, pacientes e familiares, para que possam conhecer o que a $\mathrm{PNH}$ propõe para as unidades hospitalares em benefício do paciente e/ ou familiar.

Nesse contexto, vale salientar a gestão participativa ou cogestão, proposta pela $\mathrm{PNH}$, na qual trabalhadores e usuários dos serviços de saúde são incluídos e valorizados no processo saúdedoença - com mudança da cultura de atenção aos usuários e dos processos de trabalho ${ }^{11}$.

Em virtude do desconhecimento sobre a humanização hospitalar evidenciado nas falas dos participantes deste estudo, constatou-se a necessidade de socializá-la, recorrendo a informações nos meios de comunicação, nas instituições de Ensino Superior, nas instituições hospitalares, nos serviços de saúde e nas comunidades em geral.

\section{CONCLUSÃO}

Diante da discussão desenvolvida, constatou-se satisfação com a humanização da assistência à saúde em UTI; os participantes relacionaram esse processo a ações bem realizadas, comunicação eficiente, apoio mútuo e trabalho em equipe. Eles relataram ter pouco conhecimento sobre humanização hospitalar, mas a associam a aspectos como ser caridoso(a), receber o outro com cordialidade e atenção, tratálo bem e prestar assistência à saúde com foco na eficácia e eficiência. Tudo isso ruma ao encontro da humanização hospitalar. Assim, as falas dos familiares de pacientes internados em UTI rumam ao encontro da humanização hospitalar em nosso meio.

Percebe-se a necessidade de maior envolvimento das equipes de enfermagem com os familiares, 
apresentando-se com frequência e discorrendo sobre suas funções com clareza no próprio ambiente de trabalho, visto que os familiares de pacientes internados em UTI não conseguem identificar os cuidados de enfermagem de modo espontâneo nesse cenário. As equipes de enfermagem podem aprimorar a visão global da população que busca os serviços de saúde por meio de novos comportamentos, novas atitudes e de uma postura ativa - pode-se dizer que a comunicação é fundamental para aumentar a qualidade dos serviços de saúde, com base em princípios, que envolvem a própria percepção dos aspectos envolvidos no processo saúde-doença.

Portanto, os familiares se mostram satisfeitos com a humanização da assistência em UTI, mas carecem de educação em saúde para compreender plenamente a humanização hospitalar. Isso exige que os profissionais da saúde saibam tanto lidar com a variedade de tarefas de seu trabalho cotidiano como gerir a comunicação com os familiares de pacientes hospitalizados.

\section{CONTRIBUIÇÃO DAS AUTORAS}

Elisângela de Jesus Macêdo Araújo contribuiu com a realização da pesquisa e o delineamento e a redação do manuscrito. Keila Maria de Azevedo Ponte e Lívia Mara de Araújo contribuíram com a redação do manuscrito. Maria Sinara Farias contribuiu com a revisão crítica do manuscrito.

\section{REFERÊNCIAS}

1. Duarte NE, Ferreira MA, Lisboa MTL. A dimensão prática do cuidado de enfermagem: representações sociais de acadêmicos de enfermagem. Esc Anna Nery Rev Enferm [serial on the internet]. 2012 [cited 2016 Feb 22];16(2):227-33. Available from: http:// www.scielo.br/pdf/ean/v16n2/03.pdf

2. Nascimento WSM, Silva LCC, Dias MSA, Brito MCC, Oliveira Neto JG. Cuidado da equipe de enfermagem na emergência pediátrica: revisão integrativa. Sanare (Sobral, Online) [serial on the internet]. 2017 [cited 2019 May 8];16(1):90-9. Available from: file:///D:/1099-2654-1-SM.pdf

3. Barbosa LR, Melo MRAC. Relações entre qualidade da assistência de enfermagem: revisão integrativa da literatura. Rev Bras Enferm [serial on the internet]. 2008 [cited 2016 Dec 8];61(3):366-70. Available from: http://www.scielo.br/pdf/reben/ $\underline{v 61 n 3 / a 15 v 61 n 3 . p d f}$
4. Camponogara $S$, Santos TM, Seiffert MA, Alves CN. 0 cuidado humanizado em unidade de terapia intensiva: uma revisão bibliografica. Rev Enferm UFSM [serial on the internet]. 2011 [cited 2016 Feb 23];1(1):124-32. Available from: https://periodicos. ufsm.br/reufsm/article/view/2237/1520

5. Brasil. Formação e intervenção. Brasília (DF): Ministério da Saúde; 2010. (Cadernos Humaniza SUS, Série B, v. 1).

6. Martins MM, Fernandes CS, Gonçalves LHT. A família como foco dos cuidados de enfermagem em meio hospitalar: um programa educativo. Rev Bras Enferm [serial on the internet]. 2012 [cited 2016 Nov 29];65(4):685-90. Available from: http://www. scielo.br/pdf/reben/v65n4/a20v65n4.pdf

7. Minayo MCS. Pesquisa social: teoria, método e criatividade. 29. ed. Petrópolis (RJ): Vozes; 2010. (Coleção Temas Sociais).

8. Félix TA, Ferreira FV, Oliveira EN, Eloia SC, Gomes BV, Eloia SMC. Prática da humanização na visita em unidade de terapia intensiva. Revista Enfermagem Contemporânea [serial on the internet]. 2014 [cited 2019 May 22];3(2):143-53. Available from: https:// www5.bahiana.edu.br/index.php/enfermagem/ article/view/381/342

9. Pelazza BB, Simoni RCM, Freitas EGB, Silva $B R$, Silva MJP. Visita de enfermagem e dúvidas manifestadas pela família em unidade de terapia intensiva. Acta Paul Enferm [serial on the internet]. 2015 [cited 2016 Nov 23];28(1):60-5. Available from: http://www.scielo.br/pdf/ape/v28n1/19820194-ape-028-001-0060.pdf

10. Ribeiro HSI, Silveira MGCC. Humanização hospitalar no Sistema Único de Saúde. Revista Interdisciplinar Ciências e Saúde [serial on the internet]. 2015 [cited 2016 Nov 23];2(3):19-24. Available from: http://www.ojs.ufpi.br/index.php/ rics/article/view/2040/2316

11. Chernicharo IM, Silva FD, Ferreira MA. Caracterização do termo humanização na assistência por profissionais de enfermagem. Esc Anna Nery Rev Enferm [serial on the internet]. 2014 [cited 2016 Nov 23];18(1):156-62, Available from: http://www.scielo.br/pdf/ean/v18n1/1414-8145ean-18-01-0156.pdf
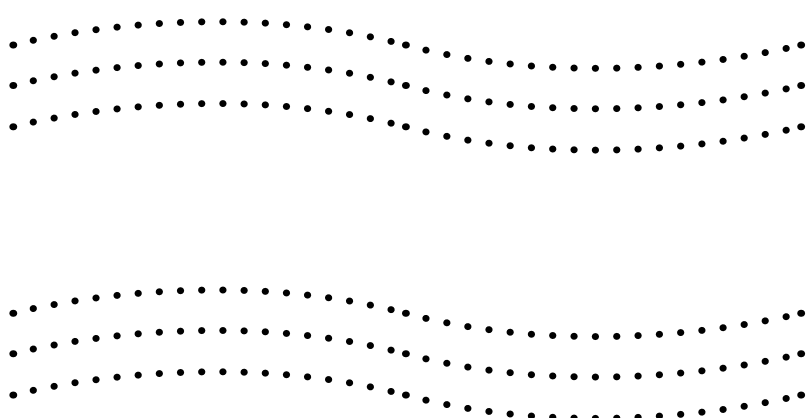